\title{
The role of the $\beta 5-\alpha 11$ loop in the active-site dynamics of acylated penicillin-binding protein A from Mycobacterium tuberculosis
}

\author{
Alena Fedarovich ${ }^{1}$, Robert A. Nicholas ${ }^{2}$, and Christopher Davies ${ }^{1, *}$ \\ ${ }^{1}$ Department of Biochemistry \& Molecular Biology, Medical University of South Carolina, \\ Charleston, SC \\ ${ }^{2}$ Departments of Pharmacology and Microbiology and Immunology, University of North Carolina \\ at Chapel Hill, Chapel Hill, NC
}

\begin{abstract}
Penicillin-binding protein A (PBPA) is a class B penicillin-binding protein that is important for cell division in M. tuberculosis. We have determined a second crystal structure of PBPA in apo form and compared it with an earlier structure of the apo enzyme. Significant structural differences in the active site region are apparent, including increased ordering of a $\beta$-hairpin loop and a shift of the SxN active site motif such that it now occupies a position that appears catalytically competent. Using two assays, including one that uses the intrinsic fluorescence of a tryptophan residue, we have also measured second-order acylation rate constants for the antibiotics, imipenem, penicillin $\mathrm{G}$ and ceftriaxone. Of these, imipenem, which has demonstrable antitubercular activity, shows the highest acylation efficiency. Crystal structures of PBPA in complex with the same antibiotics were also determined and all show conformational differences in the $\beta 5$ - $\alpha 11$ loop near the active site, but these differ for each $\beta$-lactam and also for each of the two molecules in the crystallographic asymmetric unit. Overall, these data reveal the $\beta 5-\alpha 11$ loop of PBPA as a flexible region that appears important for acylation and provide further evidence that PBPs in apo form can occupy different conformational states.
\end{abstract}

\section{Keywords \\ penicillin-binding proteins; acylation; $\beta$-lactam antibiotics; X-ray crystallography}

\section{INTRODUCTION}

Penicillin-binding proteins (PBPs) are enzymes responsible for the final stages of cell-wall formation in bacteria and are the molecular targets for $\beta$-lactam antibiotics ${ }^{1}$. Essential PBPs function as transpeptidases and catalyze the formation of cross-links between adjacent peptides in peptidoglycan, while most non-essential PBPs are carboxypeptidases or

\footnotetext{
(C) 2012 Elsevier Ltd. All rights reserved.

*To whom correspondence should be addressed: Christopher Davies: Dept. of Biochemistry and Molecular Biology, 173 Ashley Ave., Charleston, SC 29425. davies@ musc.edu. Tel: (843) 792 1468. Fax (843) 7928568.

ACCESSION NUMBERS: Coordinates and structure factors have been deposited in the Protein Data Bank with accession numbers apo, 3UN7; imipenem, 3UPN; penicillin G,3UPO and ceftriaxone, 3UPP.

Publisher's Disclaimer: This is a PDF file of an unedited manuscript that has been accepted for publication. As a service to our customers we are providing this early version of the manuscript. The manuscript will undergo copyediting, typesetting, and review of the resulting proof before it is published in its final citable form. Please note that during the production process errors may be discovered which could affect the content, and all legal disclaimers that apply to the journal pertain.
} 
endopeptidases. The hallmarks of these enzymes are three conserved motifs in the active site: the SxxK motif, which contains the serine nucleophile, the SxN motif, and the KTG motif $^{2}$. $\beta$-lactam antibiotics inhibit these enzymes by forming long-lived covalent complexes that occupy the active site and prevent binding of the peptide substrate.

Although $\beta$-lactam antibiotics have been used successfully for treatment of both Gramnegative and Gram-positive bacterial infections, they are considered to be clinically ineffective against Mycobacterium tuberculosis $(M t b)$. One reason for their low efficacy is that $M t b$ expresses the $\mathrm{BlaC} \beta$-lactamase, which hydrolyzes these antibiotics before they reach the PBP target ${ }^{3}$. Several lines of evidence, however, suggest it is premature to discount the value of $\beta$-lactams for treatment of tuberculosis. Deletion of the gene encoding $\mathrm{BlaC}$ increases sensitivity of $M t b$ to $\beta$-lactams ${ }^{4}$ and, in combination with inhibitors of $\beta$ lactamase, $\beta$-lactams show bactericidal activity against numerous strains of $M$. tuberculosis $(M t b)$, including multidrug-resistant (MDR) strains ${ }^{5}$. Moreover, a combination of amoxicillin and clavulanate shows some efficacy in patients with tuberculosis ${ }^{6}$, and meropenem in combination with clavulanate is potent against both aerobically and anaerobically grown strains of $M t b$, as well as laboratory strains of MDR and XDR $M t b^{7}$, and improves survival of mice infected with $M t b \mathrm{H} 37 \mathrm{Rv}^{8}$. Finally, imipenem alone exhibits antimycobacterial activity in both a mouse model of $M t b$ infection and in humans ${ }^{9}$. Despite the evidence that PBPs have strong potential as targets for anti-tubercular agents, there is currently an absence of structural data for the interaction of Mtb PBPs with $\beta$-lactams.

Recently, we solved the crystal structure of PBPA from $M t b$ strain H37Rv in apo form ${ }^{10}$. PBPA is a Class B-type PBP ${ }^{11}$ and, although it is not essential for cell growth ${ }^{12}$, deletion of the pbpA gene in M. smegmatis leads to reduced cell growth, defective cell morphology, and impairment of cell division ${ }^{13}$. PBPA is also of interest as it appears to be phosphorylated by $\mathrm{PknB}^{13}$, a serine/threonine protein-kinase whose gene is encoded in the same operon as pbpa. Overall, the fold of PBPA is typical for PBPs, but it has a relatively compact N-terminal domain (NTD) and an unusual conformation of the SxN active site motif and the $\beta 5-\alpha 11$ loop, which appears to restrict access to the active site, suggests that conformational changes are necessary for the protein to undergo acylation ${ }^{10}$. Another interesting feature of the crystal structure is a disulphide bridge between the SxN motif ( $\mathrm{SCN}$ in PBPA) and a partially ordered region adjacent to the active site (referred to as the $\beta$-hairpin region).

Here, we present a second crystal structure of PBPA in apo form that, when compared with the first crystal structure, exhibits a more conventional conformation for the SxN motif and the $\beta$-hairpin region is now ordered. We also determined crystal structures of acylated complexes of PBPA with penicillin G, ceftriaxone and imipenem and compared these with the second apo form. Major conformational changes in the $\beta 5-\alpha 11$ loop are apparent in these structures, which differ according to the specific $\beta$-lactam and also vary between molecules in the asymmetric unit of the crystal structures. Second-order rate constants for the acylation of PBPA by the same antibiotics were obtained using intrinsic fluorescence measurements and SDS-PAGE-binding assays, and these are correlated with the crystal structures. Overall, these data reinforce the notion that PBPs in apo form can adopt multiple conformations ${ }^{14}$ and also reveal the $\beta 5-\alpha 11$ loop as a flexible region of the active site that can adopt a variety of conformations in the acylated state of PBPA.

\section{RESULTS}

\section{Crystal structure of a second form of PBPA}

The protein used to solve the previous structure of PBPA contained the $\mathrm{His}_{6}$ tag used for purification ${ }^{10}$. The striking features of that structure were disorder in a $\beta$-hairpin region that 
lies between the SxxK and SxN motifs, apparent blockage of the active site by the $\beta 5-\alpha 11$ loop and an unusual architecture of the SxN motif that did not appear catalytically competent. Whether by crystal soaking experiments or co-crystallization, structures of the His-tagged protein as acylated complexes with $\beta$-lactams could not be obtained. For the present study, the $\mathrm{His}_{6}$ tag was removed from PBPA by cleavage with TEV protease and the resulting protein was purified and crystallized. Crystals were obtained with a very similar crystallization condition as the previous apo structure and exhibited a similar hexagonal morphology. Diffraction analysis revealed that the crystals belong to the same space group $\left(\mathrm{P}_{1}\right)$ with the same $a(=b)$ cell dimension, but the $c$ cell dimension had increased from 92 to $98 \AA$. As before, there are two molecules in the asymmetric unit, A and B.

Data extending to $2.0 \AA$ were collected from these crystals and the structure was solved by molecular replacement using the previous apo structure of PBPA as a search model (Table I), which had been solved to $2.05 \AA$ resolution ${ }^{10}$. When compared to the previous structure, the new structure of the untagged protein exhibits some interesting differences (Fig. S1). One is the ordering of a region in the N-terminal domain of molecule A that exhibited disorder in the His-tagged structure, but now forms a helix ( $\alpha 4 n$, residues 116-125) and an extension to $\beta 6$ n, together with connecting loop (see ${ }^{10}$ for secondary structure assignments). The same region in molecule $\mathrm{B}$ also exhibits increased order, but part of $\alpha 4 \mathrm{n}$ and the connecting loop to $\beta 6$ n remain disordered. Also in molecule B, residues 371-385, which form a $\beta$-hairpin ( $\beta 2 \mathrm{~h}-\beta 2 \mathrm{i}$ ) emanating from the TPase domain, are now ordered compared to the first apo structure.

The most significant differences between the two apo structures of PBPA occur in the active site region, most particularly in the SxN motif and in a loop above it that we term the $\beta$ hairpin region (Fig. 1). The shift in the SxN active site motif is such that it now exhibits a more conventional conformation when compared to other PBPs and one that is more likely to represent the functional state of the enzyme. In the structure of the tagged protein, Ser281 of the SxN motif projected away from the active site and appeared to occupy a conformation that was not catalytically competent. In the new structure, the SxN motif now occupies a position closer to the active site and the side chain of Ser281 projects between Lys 225 of the SxxK motif and Lys424 of the KTG motif (Fig. 2). The shift of this residue is substantial: the $\gamma$ oxygen of the side chain has moved $5.4 \AA$ in molecule A and $3.9 \AA$ in molecule B. In addition, it is interesting to note that the side chain of Ser222, the active site nucleophile, has rotated to form a hydrogen bond with Lys 225 in molecule B of the structure, but not in molecule A, indicating variability in the position of this residue in the same PBP.

The most likely explanation for the dramatic shift in the SxN motif is a crystallographic one and relates to increased ordering of the $\beta$-hairpin region that lies above it (as viewed in Fig. 1). Whereas a number of residues could not be modeled for the $\beta$-hairpin region in either molecule of the asymmetric unit in the structure of the tagged protein due to weak or absent electron density, all residues in this region are now completely ordered in both molecules of the new structure and the main chain B factors in this region are all below $50 \AA^{2}$. A disulphide bridge between Cys282 (the " $x$ " of the SxN motif) and Cys266 (in the $\beta 2 c-\beta 2 d$ loop) that connects the two regions provides a direct mechanism for the $\beta$-hairpin region to influence the structure of the $\mathrm{SxN}$ motif. Other contacts that link the two regions are a hydrogen bond between the side chain of Asn283 and the carbonyl of Glu259, and another between Lys 280 and the carbonyl of Cys266. In turn, increased order of the $\beta$-hairpin region may have resulted from new crystal contacts that were absent in the previous structure due to disorder. In molecule A, this region packs against the $\alpha 9-\beta 3$ loop of molecule B and may be responsible for disordering that is observed in the latter. The crystal contact for the same region in molecule $B$ is slightly different because it packs against the $\beta 4-\beta 5$ loop in addition 
to the $\alpha 9-\beta 3$ loop of a neighboring molecule $\mathrm{A}$. In contrast to molecule $\mathrm{B}$, there is no disordering the $\alpha 9-\beta 3$ loop of molecule A.

\section{Second-order rates constants of acylation for $\beta$-lactams}

PBPA contains three tryptophans, one of which (Trp442) is close to the active site. To test whether intrinsic tryptophan fluorescence could serve as a probe to monitor the covalent binding of $\beta$-lactams, PBPA was excited at $295 \mathrm{~nm}$ and a peak at $332 \mathrm{~nm}$ was observed in the intrinsic fluorescence emission spectrum (Fig. 3). Upon the addition of increasing concentrations of penicillin $\mathrm{G}$, the amplitude of the emission peak both decreased and shifted to longer wavelengths (maximum shift was to $350 \mathrm{~nm}$ ), suggesting that a conformational change in the protein occurs upon acylation with $\beta$-lactams. A similar concentration-dependent change in peak wavelength and amplitude was also observed upon similar incubation with ceftriaxone, imipenem, or Bocillin-FL, a derivative of penicillin $\mathrm{V}$ that contains a BODIPY fluorophore ${ }^{15}$. Time-dependent changes in intrinsic fluorescence were then used to calculate pseudo-first-order rate constants of acylation for each antibiotic and are shown plotted against antibiotic concentration (Fig. 4). These varied linearly and the slopes of the lines correspond to the second-order rate constants of acylation $\left(k_{2} / K_{S}\right)$ at subsaturating concentrations of antibiotic (Table II).

The second order rate constant for acylation of PBPA by Bocillin-FL was also determined by an SDS-PAGE binding assay and found to be essentially identical to the value determined by intrinsic fluorescence. The $k_{2} / K_{S}$ value for Bocillin-FL was then used to derive $k_{2} / K_{S}$ values for penicillin G, ceftriaxone and imipenem from $\mathrm{IC}_{50}$ values measured in an SDS-PAGE competition assay (Table II; see Methods for details). The $k_{2} / K_{S}$ values derived from both methods were in excellent agreement for all of the $\beta$-lactams. Bocillin-FL exhibited the highest rate of acylation, followed by imipenem, penicillin $\mathrm{G}$ and ceftriaxone.

\section{Crystal structures of acylated complexes}

In order to determine the effects of acylation on the structure of PBPA, crystal structures of acylated complexes of PBPA with imipenem, penicillin $\mathrm{G}$ or ceftriaxone were determined (Table I), and each is described below.

Imipenem structure-The structure of the acylated complex of PBPA with imipenem was solved at $2.2 \AA$ resolution. In both molecules of the asymmetric unit, the $|F o|-|F c|$ electron density corresponding to imipenem is well resolved except for the R2 side chain (Fig. 5A). This is reflected in a difference of mean B factor between R2 group and the rest of the antibiotic of $4.5 \AA^{2}$ for molecule A and $7.2 \AA^{2}$ for molecule B. In both molecules of the asymmetric unit, the $\mathrm{R} 2$ group of imipenem is positioned between $\beta 3$ and the $\beta 5-\alpha 11$ loop, and its apparent flexibility may be due to the absence of hydrogen bonding that would tether it to the enzyme. A similar weakness in density for the R2 substituent of imipenem was also observed in a complex with P. aeruginosa PBP $3{ }^{16}$. Interestingly, the $\beta 5-\alpha 11$ loop is disordered in molecule A of the crystal structure, which might correlate with the weaker density observed for the R2 group of imipenem in this molecule, whereas in molecule B, density around the $\mathrm{R} 2$ group is more evident and the $\beta 5-\alpha 11$ loop is ordered (see below).

Hydrogen-bonding interactions involving imipenem are broadly similar in both molecules of the PBPA structure, but there are differences (Fig. 6A). In molecule A, the acyl linkage carbonyl oxygen is close to the amide nitrogens of Ser222 and Thr427 (the oxyanion hole), but is only within hydrogen bonding distance of Ser222 (2.7 $⿱$ A) and not Thr427 (3.8 $)$ ). In molecule $\mathrm{B}$, the carbonyl of the acyl linkage forms hydrogen bonds with both amide nitrogens (3.2 and 2.6 $\AA$, respectively). The slightly different position of the ring carboxylate in both molecules also impacts hydrogen bonds. In molecule A, one oxygen of the ring 
carboxylate (O32) is $2.5 \AA$ from the $\gamma$ oxygen of Thr 425 of the KTG motif and the other (O31) contacts a water molecule. In molecule B, though, O31 hydrogen bonds with the $\gamma$ oxygen of Thr425 (2.5 $\AA$ ) and $\mathrm{O} 32$ is within hydrogen bonding distance of the amide nitrogen of Gly426 ( $2.8 \AA$ ). Finally, the slightly different position of the $6 \alpha$-hydroxyethyl group in each molecule means that the hydroxyl forms a hydrogen bond with the carboxamide nitrogen of Asn283 in molecule B $(2.8 \AA$ to the oxygen and $2.7 \AA$ to the nitrogen), whereas it is beyond hydrogen-bonding distance in molecule A. By contrast, a potential hydrogen bond between the ring nitrogen of imipenem and the hydroxyl of Ser281 (of the SxN motif) is essentially the same in both molecules (3.0 and 3.2 $\AA$ for molecule A and $\mathrm{B}$, respectively).

Penicillin G-The acylated complex of PBPA with penicillin G was determined at $2.3 \AA$ resolution. The density corresponding to penicillin $\mathrm{G}$ is well resolved in both molecules of PBPA, except that the phenyl ring of the R1 side chain exhibits relatively weaker density in the $|F o|-|F c|$ electron density map, especially in molecule B where its exact position is uncertain (Fig. 5B). Compared to the imipenem complex, the hydrogen bonding involving penicillin $\mathrm{G}$ is essentially the same in both molecules of the asymmetric unit. The hydrogenbonding distances between the carbonyl oxygens and the respective amide nitrogens of Ser222 and Thr427 are all the same (2.9 $\AA$ )(Fig. 6B). In addition, the carbonyl of the R group of penicillin G forms hydrogen bonds with Asn283 (2.6 and $2.5 \AA$ in molecule A and $\mathrm{B}$, respectively), similar to the interaction of the $6 \alpha$-hydroxyethyl moiety of imipenem (in molecule B). Finally, the ring nitrogen is within hydrogen-bonding distance of the Ser281 hydroxyl in both molecules ( 3.1 and $3.2 \AA$, respectively). Curiously, there is a surprising difference between the two molecules of the asymmetric unit involving an interaction made by the $\beta$-lactam carboxylate. As for the imipenem complex, an interaction between one of the oxygens of the ring carboxylate (O12) and hydroxyl of Thr425 is present, but the distance is much smaller ( $2.4 \AA$ in molecule $\mathrm{A}$ and $2.6 \AA$ in molecule $\mathrm{B})$. This close interaction is also observed in the complex with ceftriaxone (see below). The interaction made by the other oxygen (O13), however, differs between the two molecules. In molecule $\mathrm{B}$, it is directly hydrogen bonded with the amide nitrogen of Gly472 of the $\beta 5$ - $\alpha 11$ loop, but in molecule A, there is density between the two atoms, consistent with an intervening water molecule. The marked difference in conformation of the $\beta 5-\alpha 11$ loop in the two molecules (see below) may be related to this alteration in hydrogen bonding.

Ceftriaxone-The complex with ceftriaxone was solved at $2.4 \AA$ resolution and most of the ceftriaxone fits well into the $F o|-| F c \mid$ electron density map in the both molecules of the asymmetric unit, including the R1 group. In both molecules, however, the methylester group is not visible (Fig. 5C). In a situation similar to the complex with imipenem, hydrogenbonding interactions involving the acyl linkage carbonyl differ between molecules in the asymmetric unit. In molecule A, the carbonyl oxygen is $4.1 \AA$ from the amide nitrogen of Thr427, whereas the corresponding distance in molecule B is $2.8 \AA$. Likewise, the distance between the carbonyl and amide nitrogen of Ser222 is shorter in molecule A compared to molecule B ( 2.6 vs. $3.0 \AA$ ). The close interaction between one oxygen of the ring carboxylate and the $\gamma$ hydroxyl of Thr425 is present $(2.4 \AA$ in molecule $\mathrm{A}$ and $2.6 \AA$ in molecule $\mathrm{B})$. The other oxygen is within hydrogen bonding distance $(3.0 \AA)$ of the amide nitrogen of Gly472 in molecule B, but not in molecule A ( $4.5 \AA$ ). Unlike the complex with penicillin $\mathrm{G}$, an intervening water between these groups is not observed in the electron density. Like in the penicillin G complex, the R1 carbonyl and Asn283 form a potential hydrogen bond ( 2.6 and $2.8 \AA$, respectively for molecule A and B). Hydrogen bonds between the ring nitrogen and the Ser281 hydroxyl, however, are unlikely ( $3.6 \AA$ in both molecules) and this difference is likely to the six-membered dihydrothiazine ring present in cephalosporins compared to the five-membered thiazolidine ring in penicillin. Finally, in 
molecule B, the amino group of 2-amino-4-thiaazolyl ring of ceftriaxone is $3.3 \AA$ from the carbonyl of Ala469, which is the only interaction observed in the three acylated structures specific to an $\mathrm{R}$ group. The altered architecture of the $\beta 5-\alpha 11$ loop precludes a similar interaction occurring in molecule A.

\section{Conformational changes associated with acylation}

To determine what conformational changes occur as a result of acylation by $\beta$-lactam antibiotics, the three acylated structures were compared to that of apo PBPA solved from untagged protein (because this crystal form is isomorphous with the crystals used to generate the structures of acylated complexes). A superimposition of the backbones of the apo structure and the three structures of the acylated complexes shows that all structural differences map to the active site region of the enzyme (see supplemental data, Fig. S2), but differ according to the specific $\beta$-lactam and molecule in the asymmetric unit. The biggest differences occur in the $\beta 5-\alpha 11$ loop. In molecule A, this loop has shifted similarly in the complexes with penicillin $\mathrm{G}$ and ceftriaxone, and has become disordered in the imipenem complex, compared to the apo structure (Fig. 7A). In molecule B, this loop is structured in all acylated complexes, but each has a different conformation (Fig. 7B). Since this loop appears to block the active site in both apo structures of PBPA, large changes in its structure were anticipated.

By comparison, other conformational differences between acylated and apo forms of PBPA are lesser in magnitude. One of these occurs in the $\beta 3-\beta 4$ loop, which has shifted slightly in the complexes with penicillin $\mathrm{G}$ and ceftriaxone compared to the apo structure (maximum $\mathrm{C} \alpha-\mathrm{C} \alpha$ carbon shifts in the loop are 1.2 and $1.3 \AA$ for ceftriaxone and penicillin $\mathrm{G}$ complexes, respectively). Interestingly, the $\beta 3-\beta 4$ loop is disordered in both molecules of the imipenem complex structure, but why imipenem should impact this loop when its large R2 side chain points in the opposite direction is not clear. There is also a slight outward shift of the $\beta 2 \mathrm{c}-\beta 2 \mathrm{~d}$ loop in the three acylated structures with respect to the apo structure. The biggest changes occur in Tyr261 and Gly262 (the closest residues to the active site), with $\mathrm{C} \alpha$-C $\alpha$ carbon shifts up to $2 \AA$ observed in the imipenem- and ceftriaxone-acylated structures and $\sim 1 \AA$ in the penicillin complex. Within the core of the active site itself, changes are minimal and the positions of the three conserved motifs are essentially the same in all structures. For example, in molecule B of the asymmetric unit, the RMS deviations for main chain atoms of the active site residues between acylated and apo structures range from 0.2-0.3 $\AA$, compared to $0.6-0.7 \AA$ for the whole molecule. For molecule $A$, the range of RMSD values is $0.35-0.48 \AA$ for the active site residues and $0.4-1.2 \AA$ for the whole molecule. The disulphide bridge that connects the $\beta$-hairpin region with the $\mathrm{SxN}$ motif is also unchanged. It is also worth noting that Trp442, the presumed probe for intrinsic fluorescence, and its immediate environs are conformationally unchanged in all structures.

\section{DISCUSSION}

Following our structure determination of PBPA in apo form, which showed the active site apparently blocked by the $\beta 5$ - $\alpha 11$ loop and an unusual conformation of the SxN motif ${ }^{10}$, the present study sought to examine the kinetic and structural behavior of PBPA in response to acylation by $\beta$-lactam antibiotics in expectation of major conformational shifts. We obtained a second crystal structure of PBPA in apo form from a protein construct lacking the $\mathrm{His}_{6}$ purification tag, which showed several differences in the active site compared to the previous structure, and this crystal form enabled us to solve acylated structures of PBPA. These data are now discussed in light of possible dynamic behavior of the active site of PBPA and of conformational changes that occur in response to acylation by $\beta$-lactams. 
To assess the reactivity of PBPA toward antibiotics, we measured the acylation rates for several $\beta$-lactams using two assays. One took advantage of the intrinsic tryptophan

fluorescence signal in the protein, presumably Trp422 that is located near the active site, that decreases emission upon acylation of the protein by $\beta$-lactam antibiotics, and the other was an SDS-PAGE-based competition assay using the fluorescent penicillin, Bocillin-FL ${ }^{17}$. The values provided by the two methods are strikingly consistent and validate the use of intrinsic fluorescence to measure activity of PBPs when made possible by a suitably positioned tryptophan residue. Compared to some other PBPs, e.g. PBP 2 from N. gonorrhoeae or PBP 2x of $S$. pneumoniae, both of which exhibit acylation rates for penicillin $\mathrm{G}$ above 50,000 $\mathrm{M}^{-1} \mathrm{sec}^{-1} 18,19$, PBPA shows only moderate sensitivity to $\beta$-lactams, but it is comparable to others, e.g. S. pneumoniae PBP1b, which also exhibits similarly low rates with penicillin ${ }^{20}$.

Of the four $\beta$-lactams tested, the order of $k_{2} / K_{S}$ acylation rates, highest to lowest, was Bocillin-FL > imipenem > penicillin $\mathrm{G}>$ ceftriaxone. The relatively higher activity with imipenem is interesting as penems have demonstrable efficacy against $M$. tuberculosis, including a mouse model of TB infection and in clinical treatment ${ }^{7,9,21}$. For other PBPs where rates of acylation have been measured against a series of $\beta$-lactams, different patterns are observed. For example, whilst imipenem reacts with $S$. pneumoniae PBP $2 \mathrm{x}$ at a faster acylation rate than penicillin $\mathrm{G}$, and some cephalosporins (e.g. cefotaxime) react at higher rates than both imipenem and penicillin, other cephalosporins (e.g. cephalexin) exhibit acylation rates lower than all three ${ }^{22}$. For $N$. gonorrhoeae PBP 2, two cephalosporins (ceftriaxone and cefixime) both exhibit markedly higher rates of acylation compared to penicillin $\mathrm{G}^{18}$, which is opposite to PBPA. Finally, for E. coli PBP 5, the corresponding order of acylation rates is cefoxitin (a cephalosporin) > imipenem > cloxacillin (a penicillin) ${ }^{23}$. Two points emerge from such data: 1 ) the acylation rates vary for the same antibiotic against different PBPs, and 2) rates for different antibiotics directed against the same PBP also vary and appear specific for that target.

It is difficult to explain the relative order of acylation rates based on the structural data especially since, in all complexes, there is a paucity of specific interactions between the R1 or R2 groups and the enzyme, and because differences are observed between the two molecules of the asymmetric unit. In fact, whilst imipenem has the highest rate of acylation of the three antibiotics against PBPA, its R2 group is the least ordered of the $\beta$-lactam side chains. Conversely, ceftriaxone has the lowest rate of acylation and yet its R1 group shows the most ordered binding. The greater activity of imipenem may be due to its $\mathrm{R} 2$ group projecting toward the $\beta 5-\alpha 11$ loop, whereas the R1 groups of penicillin $\mathrm{G}$ and ceftriaxone are directed toward the $\beta 2 \mathrm{c}-\beta 2 \mathrm{~d}$ loop on the opposite side of the active site. Hence, it is possible that acylation rates are lowered by steric repulsion involving these R1 groups, which would not occur with imipenem because it lacks an R1 group.

The apparent disorder in the R1 or R2 groups of the $\beta$-lactams in the crystal structures is unexpected and contrasts with some complexes of other PBPs where electron densities for these regions are well defined ${ }^{24-28}$. Our findings are similar, however, to those we reported for E. coli PBP 5, where, in crystal structures of acylated complexes, electron density for the $\mathrm{R} 1 / \mathrm{R} 2$ substituents of various $\beta$-lactams was also consistently weak and did not correlate with acylation rates ${ }^{29}$. Such data reinforce the notion that acylated complexes of PBPs with $\beta$-lactams may only be of limited value when rationally designing new inhibitors of PBPs because these may not reveal interactions that occurred during acylation ${ }^{23}$. In support of this, crystal structures of the AmpC $\beta$-lactamase in the pre-covalent, acylated and postcovalent product state show significantly different modes of binding for cephalothin ${ }^{30}$.

One surprise in the acylation data is the nearly 3 -fold difference in rate between penicillin $\mathrm{G}$ and Bocillin-FL. The latter differs from penicillin $\mathrm{G}$ by having an ester linkage to the R1 
phenol group (because it is a derivative of penicillin V) and by containing a BODIPY fluorophore attached to the para-position of the phenol group via an intervening amide group. Either or both of these differences could be responsible for the different rates. Data collected from crystals of PBPA that had been soaked in Bocillin-FL (at different molarities and with various soak times) consistently showed evidence of acylation of Ser222 in the active site, but density beyond that was uninterpretable and none of the R1 group was visible (data not shown). One possible explanation for weak density of Bocillin-FL is that, whilst the $\beta$-lactam ring can access the serine nucleophile, the large R1 group with its pendant BODIPY group does not bind the protein and is disordered.

The previous structure of PBPA in apo form showed disorder in the $\beta$-hairpin region, a conformation for the SxN motif that did not appear catalytically competent, and an active site that appeared to be occluded by the $\beta 5-\alpha 11$ loop ${ }^{10}$. At the time, we assumed that major conformational changes would be required during reaction with $\beta$-lactams (and peptidoglycan substrate). The second structure of PBPA in apo form reported here was determined using protein devoid of its purification tag, which generated a slightly altered crystal form. This structure shows increased order in the $\beta$-hairpin region and a canonical position for the $\mathrm{SxN}$ motif, but the $\beta 5-\alpha 11$ loop remains in a similar position. The conformational differences observed between two apo forms of PBPA is reminiscent of $S$. pneumoniae PBP $1 \mathrm{~b}$ in which there are "open" and "closed" architectures involving movement of three loops near the active site in two forms of the apoenzyme ${ }^{14}$. In the case of PBPA, a new crystal contact between Gly253, which lies on the $\beta$-hairpin region between $\beta 2 \mathrm{~b}$ and $\beta 2 \mathrm{c}$, and the $\alpha 9-\beta 3$ loop of neighboring molecule may be responsible for greater ordering of the $\beta$-hairpin region and this, in turn, may alter the conformation of the SxN motif through the disulphide bridge between Cys266 and Cys282. It appears therefore that structural changes observed between apo forms are a consequence of slightly different crystal packing of the His-tagged vs. untagged forms of PBPs, and in support of this, acylation rates for the tagged versus untagged protein are essentially identical. How the tag itself influences crystallization is unclear because in both apo structures, the traceable model begins at around residue 45 and residues prior to that, including the tag in the first apo structure, are not visible.

It is notable that the $\beta$-hairpin region of PBPA was disordered in the first structure of apo PBPA but is now ordered in the new structure, because it suggests this area of the molecule is not rigid and can adopt different conformations depending on the crystallographic packing interactions of different crystal forms. This is interesting because it is broadly equivalent to a region of S. pneumoniae PBP 2x comprising residues 364-395 leading up to the SxN motif that exhibits order in the enzyme derived from the penicillin-susceptible strain R6 ${ }^{31}$, but is disordered in PBP 2x from the penicillin-resistant strain Sp328 ${ }^{32}$. This difference was suggested as the cause for lowered acylation efficiency of Sp328-PBP2x, but, because the two crystal structures were obtained from different crystal forms $\left(\mathrm{P}_{1}{ }_{1} 2_{1} 2\right.$ vs. $\left.\mathrm{P} 3_{2}\right)$, the observed structural changes could be a consequence of crystallization and unrelated to penicillin resistance. That said, this region is apparently more susceptible to proteolytic digestion in PBP2x derived from resistant strains, but not susceptible strains ${ }^{33}$, which is corroborating evidence of increased flexibility in this loop. Nevertheless, since the only difference between the two PBPA proteins is the presence of a purification tag, with no discernable effect on acylation efficiency by $\beta$-lactams, our findings urge a cautionary approach to the interpretation of purely crystallographic data.

We also observed evidence of flexibility in our three structures of PBPA in acylated form. The key element involved in these structures is the $\beta 5-\alpha 11$ loop, which has shifted or become disordered in all three acylated complexes, in agreement with the expectation that this loop would move during acylation to allow access to the active site. Interestingly, the 
loop exhibits a variety of conformations (or degrees of order) depending on the specific $\beta$ lactam, and also changes between the two molecules in the asymmetric unit. For the imipenem complex, the $\beta 5-\alpha 11$ loop is disordered in molecule $A$ and has shifted in molecule $\mathrm{B}$ (compared to the apo structure). Its position may be dictated by the $\mathrm{R} 2$ substituent of imipenem, which, in both molecules of the asymmetric unit, points toward the loop. For the complex with penicillin G, even though the $\beta 5-\alpha 11$ loop is ordered in both molecules of the asymmetric unit, there are surprising differences in its conformation depending on whether an interaction between ring carboxylate and loop is direct or involves a bridging water molecule. The $\beta 5-\alpha 11$ loop also adopts different conformations in each molecule of the ceftriaxone complex, even though the binding mode of ceftriaxone is very similar in both. The importance of the $\beta 5-\alpha 11$ loop for acylation is also highlighted by shifts in this region between apo and acylated forms of PBP3 from P. aeruginosa ${ }^{16}$ and in S. pneumoniae PBP1b ${ }^{14,20}$ and the fact that two Arg to Gln mutations in this loop in S. pneumoniae PBP1b lower second-order acylation rate constants for $\beta$-lactams by several fold ${ }^{20}$. Likewise, we have shown that mutation of Gly545 to Ser in the $\beta 5-\alpha 11$ loop in N. gonorrhoeae PBP 2 lowers the acylation rates with penicillin and expanded-spectrum cephalosporins ${ }^{18}$. Hence, the $\beta 5-\alpha 11$ loop is emerging as an important functional region for acylation of PBPs, alongside other well-established regions such as the $\beta 3-\beta 4$ loop $20,28,34,35$.

In conclusion, a second structure of Mtb PBPA in apo form provides evidence that PBPs can occupy different conformational states in solution and even within a single crystal. Kinetic measurements of the acylation efficiency of the enzyme using several $\beta$-lactams shows that PBPA reacts relatively poorly compared with other PBPs, but the highest activity with imipenem may correlate with its clinical efficacy. Conformational changes induced by acylation center on the $\beta 5-\alpha 11$ loop of the active site and are specific for each $\beta$-lactam. Overall, these data highlight the importance of the $\beta 5-\alpha 11$ loop in the active-site dynamics of PBPs and reactivity with $\beta$-lactams.

\section{MATERIALS \& METHODS}

\section{Materials}

Penicillin G, ceftriaxone, and imipenem were purchased from Sigma (St. Louis, MO), and Bocillin-FL from Invitrogen (Carlsbad, CA).

\section{Protein purification}

A soluble form of wild-type PBPA lacking its transmembrane anchor and signal sequence (residues 1-33) was cloned into pT7HTb, expressed in E. coli BL21 DE3 cells, refolded and purified by $\mathrm{Ni}^{2+}$-affinity chromatography, as described previously ${ }^{10}$. This protein contains a hexahistidine tag and intervening cleavage site for tobacco etch virus (TEV) protease. To remove the tag, the fusion protein was dialyzed against buffer A $(20 \mathrm{mM}$ Tris- $\mathrm{HCl}, \mathrm{pH} 8.0$, $0.5 \mathrm{M} \mathrm{NaCl}, 10 \%$ glycerol, $20 \mathrm{mM}$ imidazole) overnight at $4{ }^{\circ} \mathrm{C}$ and then mixed with $\mathrm{His}_{6}{ }^{-}$ tagged TEV-protease in a 50:1 ratio (w/w). PBPA was isolated from the digest by a second passage over $\mathrm{Ni}^{+2}$-affinity column (HisTrap HP, GE Healthcare) that had been equilibrated with buffer A. PBPA eluted in the flow-through and was concentrated to $9 \mathrm{mg} / \mathrm{ml}$.

\section{Kinetic analysis of the interaction of PBPA with $\beta$-lactams}

PBPs react with $\beta$-lactam antibiotics according to the kinetic scheme:

$$
\mathrm{E}+\mathrm{S} \stackrel{K s}{\longleftrightarrow} \mathrm{E} \bullet \mathrm{S} \stackrel{k_{2}}{\longrightarrow} \mathrm{E}-\mathrm{S}^{\prime} \stackrel{k_{3}}{\longrightarrow} \mathrm{E}+\mathrm{P}
$$


where E•S is the non-covalent Michaelis-Menten complex, E-S is the covalent acyl-enzyme complex, and $\mathrm{P}$ is the product after deacylation. For $\beta$-lactams, the deacylation rate is very slow and so the second-order acylation rate constant $\left(k_{2} / K_{S}\right)$, which corresponds to the initial rate of formation of covalent $\beta$-lactam adducts, is used as a measure of the potency of an antibiotic. At sub-saturating conditions, when $[\mathrm{S}]<K_{m}$, the kinetics are described by equation (1):

$$
k_{a}=v / \mathrm{E}_{\mathrm{t}}=[\mathrm{S}] k_{2} / K_{S}
$$

where [S] is the $\beta$-lactam concentration, $k_{a}$ is the pseudo-first-order rate constant of E-S formation at a given $[S]$ value, $v$ is the initial rate of formation of $E-S$, and $E_{t}$ is total enzyme concentration ${ }^{36}$. The second-order acylation rate constants for the reaction of PBPA with penicillin G, ceftriaxone, imipenem, and the fluorescent penicillin Bocillin-FL ${ }^{15}$ were determined measuring time-dependent decreases of PBPA intrinsic fluorescence and SDSPAGE binding assays.

\section{Intrinsic fluorescence}

Intrinsic fluorescence emission spectra of PBPA were recorded at $36^{\circ} \mathrm{C}$ on a Quanta Master fluorimeter (QM4, Photon Technology International, Inc., Birmingham, NJ) with fluorescence excitation at $295 \mathrm{~nm}$ and emission at $332 \mathrm{~nm}$ (slit widths of $2 \mathrm{~nm}$ and $4 \mathrm{~nm}$, respectively). For all experiments, PBPA was diluted into $2 \mathrm{ml}$ of binding buffer $(50 \mathrm{mM}$ sodium phosphate, $\mathrm{pH} 8$ ) to a final concentration of $0.5 \mu \mathrm{M}$ and then mixed with $\beta$-lactams. The time-dependent decrease of intrinsic fluorescence was recorded immediately after addition of each $\beta$-lactam in the sub-saturating concentration range of 2 to $25 \mu \mathrm{M}$. At least three independent measurements were performed at each $\beta$-lactam concentration. Data were corrected for the buffer and ligand background signals. Using equation (1), $k_{2} / K_{S}$ was derived from a plot of $k_{a}$ vs [S], where the slope of the line defines the second-order acylation rate constant.

\section{SDS-PAGE-based binding assays}

PBPA $(1 \mu \mathrm{M})$ was incubated at $36^{\circ} \mathrm{C}$ with $2.5,5,8,10$, or $12 \mu \mathrm{M}$ Bocillin-FL. Every $10-30$ sec, $20 \mu \mathrm{l}$ aliquots were removed, mixed with $5 \mu \mathrm{l}$ of SDS-PAGE sample buffer and boiled at $98^{\circ} \mathrm{C}$ for $2 \mathrm{~min}$. Acylated PBPA was then separated from free label using $10 \%$ MiniProtean TGX SDS-PAGE gels (Bio-Rad, Hercules, CA). At least two independent reactions were performed in duplicate at each antibiotic concentration. Gels were scanned using a Kodak EDAS 290 UV imaging system (Scientific Imaging Systems, Eastman Kodak, Newhaven, CT, USA), following which the gels were stained with Coomassie R-250 to confirm equivalent loading. Densitometry was performed with the ImageJ 1.37 program (National Institutes of Health, USA; http//rsb.info.nih.gov/ij/) and data points were normalized to the maximum of the fluorescence intensity (defining complete saturation of PBPA by Bocillin-FL). $k_{a}$ values were determined at each antibiotic concentration from plots of $\%$ bound versus time using nonlinear regression analysis and the $k_{2} / K_{S}$ of BocillinFL was derived from a plot of $k_{a}$ versus [S].

In the competition assay, PBPA $(1 \mu \mathrm{M})$ was incubated with $10 \mu \mathrm{M}$ Bocillin-FL in the presence of increasing concentrations $(0.05-1000 \mu \mathrm{M})$ of an unlabeled $\beta$-lactam (penicillin $\mathrm{G}$, ceftriaxone, or imipenem) in $20 \mu \mathrm{l}$ final volume at $36^{\circ} \mathrm{C}$ for $15 \mathrm{~min}$ and PBPA bound by Bocillin-FL was quantified by densitometry after SDS-PAGE. Data from four independent experiments (in duplicate for each antibiotic) were used to determine $\mathrm{IC}_{50}$ values, from which second-order acylation rate constants were derived using equation (2) ${ }^{36}$ : 


$$
\left(k_{2} / K_{S}\right)_{\mathrm{U}}=\left(k_{2} / K_{S}\right)_{\mathrm{L}}\left[\mathrm{S}_{\mathrm{L}}\right] / \mathrm{IC}_{50}
$$

where $\left(k_{2} / K_{S}\right)_{\mathrm{U}}$ is the rate constant of an unlabeled compound, $\left(k_{2} / K_{S}\right)_{\mathrm{L}}$ is the rate constant of Bocillin-FL, and $\left[\mathrm{S}_{\mathrm{L}}\right]$ is the concentration of Bocillin-FL used in the competition experiment.

All curve fitting analysis and calculations for both intrinsic fluorescence and SDS-PAGE methods were performed using GraphPad Prism version 4.00 for Windows (GraphPad Software, Inc, San Diego, CA).

\section{Crystallization, data collection and structure refinement of the new apo form and acylated complexes}

Crystals of PBPA were grown using the hanging drop vapor diffusion method at room temperature over wells containing 25-27\% PEG 3350, $0.2 \mathrm{M} \mathrm{NaCl}$ and $0.1 \mathrm{M}$ Bis-Tris pH 5.5-5.6. To generate acylated complexes, crystals were soaked in $100 \mathrm{mM}$ penicillin $\mathrm{G}$ for 3 hours or $100 \mathrm{mM}$ ceftriaxone for 2 hours. Crystals used to solve the complex with imipenem were grown over 20\% PEG 3350 and $0.2 \mathrm{M}$ potassium nitrate, and were soaked in $16 \mathrm{mM}$ of the antibiotic for 5 hours. All crystals were cryoprotected by passage through the respective mother liquor to which ethylene glycol had been added to a final concentration of $30 \%$ and then frozen in liquid nitrogen.

X-ray diffraction data were collected at the SER-CAT ID-22 beam line of the Advanced Photon Source (Argonne National Laboratory, Chicago, IL) at $1.00 \AA$ A wavelength. The crystals belong to space group $\mathrm{P} 6{ }_{1}$, with cell dimensions of $\sim a=b=122 \AA$ and $c=98 \AA$ and two molecules in the asymmetric unit. All data were processed with HKL2000 ${ }^{37}$. The starting model for refinement was the previous structure of PBPA in apo form, but stripped of all water molecules ${ }^{10}$. For each acylated structure, after an initial round of rigid-body and restrained refinement using REFMAC5 ${ }^{38},(|F o|-|F c|)$ and $2(|F o|-|F c|)$ maps were calculated and used to model the $\beta$-lactam and rebuild the protein using $\mathrm{O}^{39}$. The PRODRG server was used to generate stereochemical libraries for the refinement of each antibiotic ${ }^{40}$. The model was then improved by further rounds of manual building and REFMAC, including introduction of ordered water molecules. All atoms were refined with an occupancy of 1, including those of the antibiotics. The geometry of the four structures was validated by PROCHECK ${ }^{41}$. In all structures, residues Ser96, Ala99 and Lys340 consistently lie outside favored regions of the Ramachandran plot, but occupy excellent electron density. Some features in the electron density maps indicating binding of unknown ligands from the crystallization or soaking solutions could not be modeled.

\section{Supplementary Material}

Refer to Web version on PubMed Central for supplementary material.

\section{Acknowledgments}

This work was supported by the National Institutes of Health grants GM66861 to C.D. and AI36901 to R.A.N. Use of the Advanced Photon Source was supported by the U.S. Department of Energy, Office of Science, Office of Basic Energy Sciences, under Contract No. W-31-109-ENG-38. Data were collected at Southeast Regional Collaborative Access Team (SER-CAT) 22-ID beamline at the Advanced Photon Source, Argonne National Laboratory. Supporting institutions may be found at www.ser-cat.org/members.html. The X-ray crystallography facility used for this work is supported by the Medical University of South Carolina's Research Resource Facilities program MUSC. 


\section{Abbreviations used}

$\begin{array}{ll}\text { HMM } & \text { high molecular mass } \\ \text { LMM } & \text { low molecular mass } \\ \text { Mtb } & \text { Mycobacterium tuberculosis } \\ \text { NTD } & \text { N-terminal domain } \\ \text { PBP } & \text { penicillin-binding protein } \\ \text { RMSD } & \text { root mean square deviation } \\ \text { TPase } & \text { transpeptidase }\end{array}$

\section{References}

1. Macheboeuf P, Contreras-Martel C, Job V, Dideberg O, Dessen A. Penicillin binding proteins: key players in bacterial cell cycle and drug resistance processes. FEMS Microbiol Rev. 2006; 30:673691. [PubMed: 16911039]

2. Ghuysen JM. Serine $\beta$-lactamases and penicillin-binding proteins. Annu Rev Microbiol. 1991; 45:37-67. [PubMed: 1741619]

3. Cole ST, Brosch R, Parkhill J, Garnier T, Churcher C, Harris D, Gordon SV, Eiglmeier K, Gas S, Barry CE 3rd, Tekaia F, Badcock K, Basham D, Brown D, Chillingworth T, Connor R, Davies R, Devlin K, Feltwell T, Gentles S, Hamlin N, Holroyd S, Hornsby T, Jagels K, Krogh A, McLean J, Moule S, Murphy L, Oliver K, Osborne J, Quail MA, Rajandream MA, Rogers J, Rutter S, Seeger K, Skelton J, Squares R, Squares S, Sulston JE, Taylor K, Whitehead S, Barrell BG. Deciphering the biology of Mycobacterium tuberculosis from the complete genome sequence. Nature. 1998; 393:537-544. [PubMed: 9634230]

4. Flores AR, Parsons LM, Pavelka MS Jr. Genetic analysis of the $\beta$-lactamases of Mycobacterium tuberculosis and Mycobacterium smegmatis and susceptibility to beta-lactam antibiotics. Microbiology. 2005; 151:521-532. [PubMed: 15699201]

5. Dincer I, Ergin A, Kocagoz T. The vitro efficacy of beta-lactam and beta-lactamase inhibitors against multidrug resistant clinical strains of Mycobacterium tuberculosis. Int J Antimicrob Agents. 2004; 23:408-411. [PubMed: 15081094]

6. Chambers HF, Kocagoz T, Sipit T, Turner J, Hopewell PC. Activity of amoxicillin/clavulanate in patients with tuberculosis. Clin Infect Dis. 1998; 26:874-877. [PubMed: 9564467]

7. Hugonnet JE, Tremblay LW, Boshoff HI, Barry CE 3rd, Blanchard JS. Meropenem-clavulanate is effective against extensively drug-resistant Mycobacterium tuberculosis. Science. 2009; 323:12151218. [PubMed: 19251630]

8. Veziris N, Truffot C, Mainardi JL, Jarlier V. Activity of carbapenems combined with clavulanate against murine tuberculosis. Antimicrob Agents Chemother. 2011; 55:2597-2600. [PubMed: 21402832]

9. Chambers HF, Turner J, Schecter GF, Kawamura M, Hopewell PC. Imipenem for treatment of tuberculosis in mice and humans. Antimicrob Agents Chemother. 2005; 49:2816-2821. [PubMed: 15980354]

10. Fedarovich A, Nicholas RA, Davies C. Unusual conformation of the SxN motif in the crystal structure of penicillin-binding protein A from Mycobacterium tuberculosis. J Mol Biol. 2010; 398:54-65. [PubMed: 20206184]

11. Goffin C, Ghuysen JM. Biochemistry and comparative genomics of SxxK superfamily acyltransferases offer a clue to the mycobacterial paradox: presence of penicillin-susceptible target proteins versus lack of efficiency of penicillin as therapeutic agent. Microbiol Mol Biol Rev. 2002; 66:702-738. [PubMed: 12456788]

12. Sassetti CM, Boyd DH, Rubin EJ. Genes required for mycobacterial growth defined by high density mutagenesis. Mol Microbiol. 2003; 48:77-84. [PubMed: 12657046] 
13. Dasgupta A, Datta P, Kundu M, Basu J. The serine/threonine kinase PknB of Mycobacterium tuberculosis phosphorylates PBPA, a penicillin-binding protein required for cell division. Microbiology. 2006; 152:493-504. [PubMed: 16436437]

14. Lovering AL, De Castro L, Lim D, Strynadka NC. Structural analysis of an "open” form of PBP1B from Streptococcus pneumoniae. Protein Sci. 2006; 15:1701-1709. [PubMed: 16751607]

15. Gee KR, Kang HC, Meier TI, Zhao G, Blaszcak LC. Fluorescent Bocillins: synthesis and application in the detection of penicillin-binding proteins. Electrophoresis. 2001; 22:960-965. [PubMed: 11332764]

16. Han S, Zaniewski RP, Marr ES, Lacey BM, Tomaras AP, Evdokimov A, Miller JR, Shanmugasundaram V. Structural basis for effectiveness of siderophore-conjugated monocarbams against clinically relevant strains of Pseudomonas aeruginosa. Proc Natl Acad Sci U S A. 2010; 107:22002-22007. [PubMed: 21135211]

17. Zhao G, Meier TI, Kahl SD, Gee KR, Blaszczak LC. BOCILLIN FL, a sensitive and commercially available reagent for detection of penicillin-binding proteins. Antimicrob Agents Chemother. 1999; 43:1124-1128. [PubMed: 10223924]

18. Tomberg J, Unemo M, Davies C, Nicholas RA. Molecular and Structural Analysis of Mosaic Variants of Penicillin-Binding Protein 2 Conferring Decreased Susceptibility to ExpandedSpectrum Cephalosporins in Neisseria gonorrhoeae: Role of Epistatic Mutations. Biochemistry. 2010; 49:8062-8070. [PubMed: 20704258]

19. Jamin M, Wilkin JM, Frere JM. A new kinetic mechanism for the concomitant hydrolysis and transfer reactions catalyzed by bacterial DD-peptidases. Biochemistry. 1993; 32:7278-7285. [PubMed: 8343517]

20. Macheboeuf P, Di Guilmi AM, Job V, Vernet T, Dideberg O, Dessen A. Active site restructuring regulates ligand recognition in class A penicillin-binding proteins. Proc Natl Acad Sci U S A. 2005; 102:577-582. [PubMed: 15637155]

21. Lavollay M, Arthur M, Fourgeaud M, Dubost L, Marie A, Veziris N, Blanot D, Gutmann L, Mainardi JL. The peptidoglycan of stationary-phase Mycobacterium tuberculosis predominantly contains cross-links generated by L,D-transpeptidation. J Bacteriol. 2008; 190:4360-6. [PubMed: 18408028]

22. Jamin M, Hakenbeck R, Frere JM. Penicillin binding protein $2 \mathrm{x}$ as a major contributor to intrinsic $\beta$-lactam resistance of Streptococcus pneumoniae. FEBS Lett. 1993; 331:101-104. [PubMed: 8405385]

23. Beadle BM, Nicholas RA, Shoichet BK. Interaction energies between $\beta$-lactam antibiotics and $E$. coli penicillin-binding protein 5 by reversible thermal denaturation. Protein Sci. 2001; 10:12541259. [PubMed: 11369864]

24. Lim D, Strynadka NC. Structural basis for the $\beta$ lactam resistance of PBP2a from methicillinresistant Staphylococcus aureus. Nat Struct Biol. 2002; 9:870-876. [PubMed: 12389036]

25. Sauvage E, Herman R, Petrella S, Duez C, Bouillenne F, Frere JM, Charlier P. Crystal structure of the Actinomadura R39 DD-peptidase reveals new domains in penicillin-binding proteins. J Biol Chem. 2005; 280:31249-31256. [PubMed: 15987687]

26. Kishida H, Unzai S, Roper DI, Lloyd A, Park SY, Tame JR. Crystal structure of penicillin binding protein $4(\mathrm{dacB})$ from Escherichia coli, both in the native form and covalently linked to various antibiotics. Biochemistry. 2006; 45:783-792. [PubMed: 16411754]

27. Kawai F, Clarke TB, Roper DI, Han GJ, Hwang KY, Unzai S, Obayashi E, Park SY, Tame JR. Crystal structures of penicillin-binding proteins 4 and 5 from Haemophilus influenzae. J Mol Biol. 2010; 396:634-645. [PubMed: 19958776]

28. Sainsbury S, Bird L, Rao V, Shepherd SM, Stuart DI, Hunter WN, Owens RJ, Ren J. Crystal structures of penicillin-binding protein 3 from Pseudomonas aeruginosa: comparison of native and antibiotic-bound forms. J Mol Biol. 2011; 405:173-184. [PubMed: 20974151]

29. Nicola G, Tomberg J, Pratt RF, Nicholas RA, Davies C. Crystal structures of covalent complexes of $\beta$-lactam antibiotics with Escherichia coli penicillin-binding protein 5: toward an understanding of antibiotic specificity. Biochemistry. 2010; 49:8094-8104. [PubMed: 20726582] 
30. Beadle BM, Trehan I, Focia PJ, Shoichet BK. Structural milestones in the reaction pathway of an amide hydrolase: substrate, acyl, and product complexes of cephalothin with AmpC $\beta$-lactamase. Structure. 2002; 10:413-424. [PubMed: 12005439]

31. Gordon E, Mouz N, Duee E, Dideberg O. The crystal structure of the penicillin-binding protein 2x from Streptococcus pneumoniae and its acyl-enzyme form: implication in drug resistance. J Mol Biol. 2000; 299:477-485. [PubMed: 10860753]

32. Dessen A, Mouz N, Gordon E, Hopkins J, Dideberg O. Crystal structure of PBP2x from a highly penicillin-resistant Streptococcus pneumoniae clinical isolate: a mosaic framework containing 83 mutations. J Biol Chem. 2001; 276:45106-45112. [PubMed: 11553637]

33. Carapito R, Chesnel L, Vernet T, Zapun A. Pneumococcal $\beta$-lactam resistance due to a conformational change in penicillin-binding protein 2x. J Biol Chem. 2006; 281:1771-1777. [PubMed: 16303769]

34. Nicola G, Peddi S, Stefanova ME, Nicholas RA, Gutheil WG, Davies C. Crystal structure of Escherichia coli penicillin-binding protein 5 bound to a tripeptide boronic acid inhibitor: a role for Ser-110 in deacylation. Biochemistry. 2005; 44:8207-8217. [PubMed: 15938610]

35. Chen Y, Zhang W, Shi Q, Hesek D, Lee M, Mobashery S, Shoichet BK. Crystal structures of penicillin-binding protein 6 from Escherichia coli. J Am Chem Soc. 2009; 131:14345-14354. [PubMed: 19807181]

36. Frere, JM.; Nguyen-Disteche, M.; Coyette, J.; Joris, B. Mode of action: interaction with the penicillin-binding proteins. In: Page, MI., editor. The Chemistry of $\beta$-lactams. Chapman \& Hall; Glasgow: 1992. p. 148-196.

37. Otwinowski Z, Minor W. Processing of X-ray diffraction data collected in oscillation mode. Meths Enzymol. 1997; 276:307-326.

38. Murshudov GN, Vagin AA, Dodson EJ. Refinement of macromolecular structures by the maximum-likelihood method. Acta Cryst. 1997; D53:240-255.

39. Jones TA, Zou J-Y, Cowan SW, Kjeldgaard M. Improved methods for building protein structures in electron-density maps and the location of errors in these models. Acta Cryst. 1991; A47:110119.

40. Schuttelkopf AW, van Aalten DM. PRODRG: a tool for high-throughput crystallography of protein-ligand complexes. Acta Crystallogr D Biol Crystallogr. 2004; 60:1355-1363. [PubMed: 15272157]

41. Laskowski RA, MacArthur MW, Moss DS, Thornton JM. PROCHECK: a program to check the stereochemical quality of protein structures. J Appl Cryst. 1993; 26:283-291. 


\section{Highlights}

- Apoenzyme forms of PBPA from M. tuberculosis exhibit altered active site structures.

- Of several $\beta$-lactams tested, imipenem acylates PBPA at the highest rate.

- Acylation of PBPA by $\beta$-lactams induces large shifts in the $\beta 5-\alpha 11$ active site loop.

- PBPA exhibits dynamic behavior prior to and during acylation by $\beta$-lactams. 
A
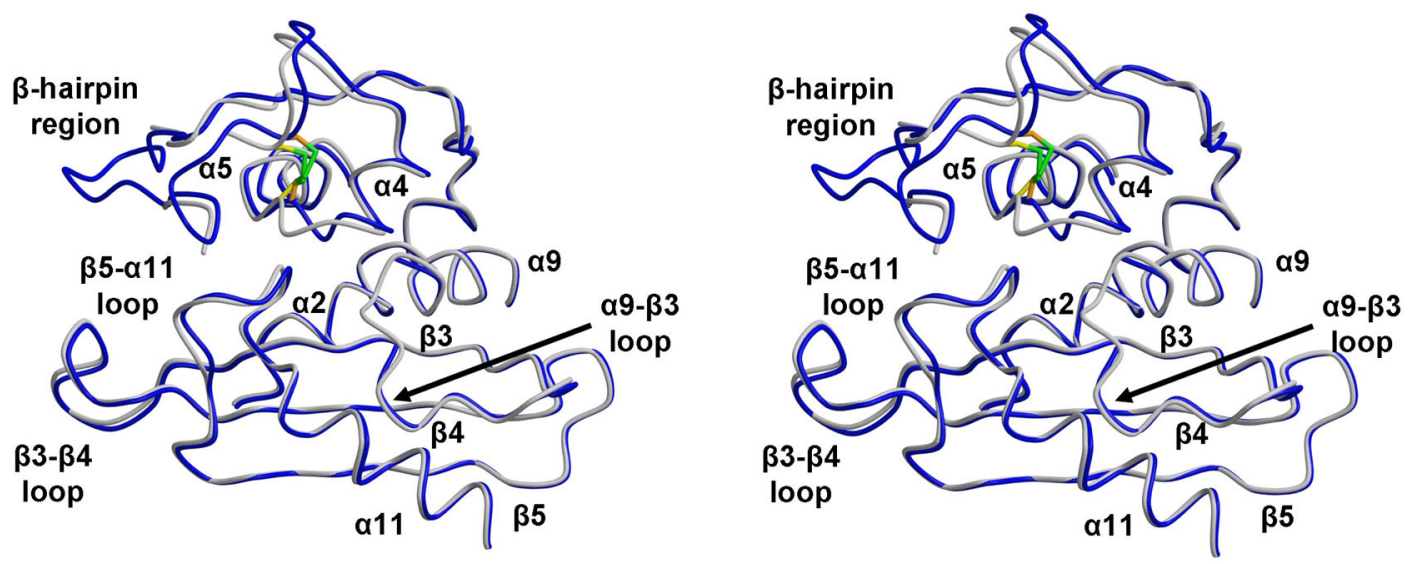

B
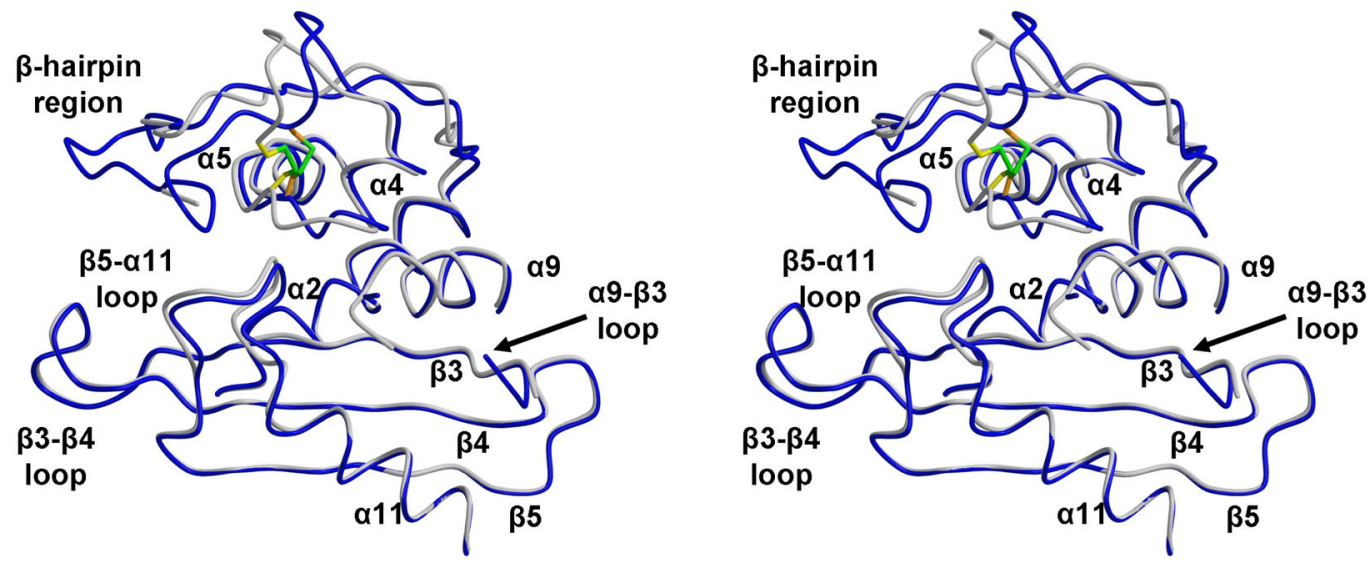

Fig. 1.

A comparison of two structures of Mtb PBPA in apo form. The new apo structure was superimposed with an apo structure determined previously ${ }^{10}$ and structural differences in the active site region are shown for both molecules in the asymmetric unit. In this stereoview, the C $\alpha$ backbone of the previous (His-tagged) apo structure is colored grey and that of the new apo structure (untagged) is blue. Elements of secondary structure are labeled and the disulfide bridge linking the $\beta$-hairpin region with the $\mathrm{SxN}$ motif is shown in green. Note the increased ordering of the $\beta$-hairpin region in the new apo structure. 
A
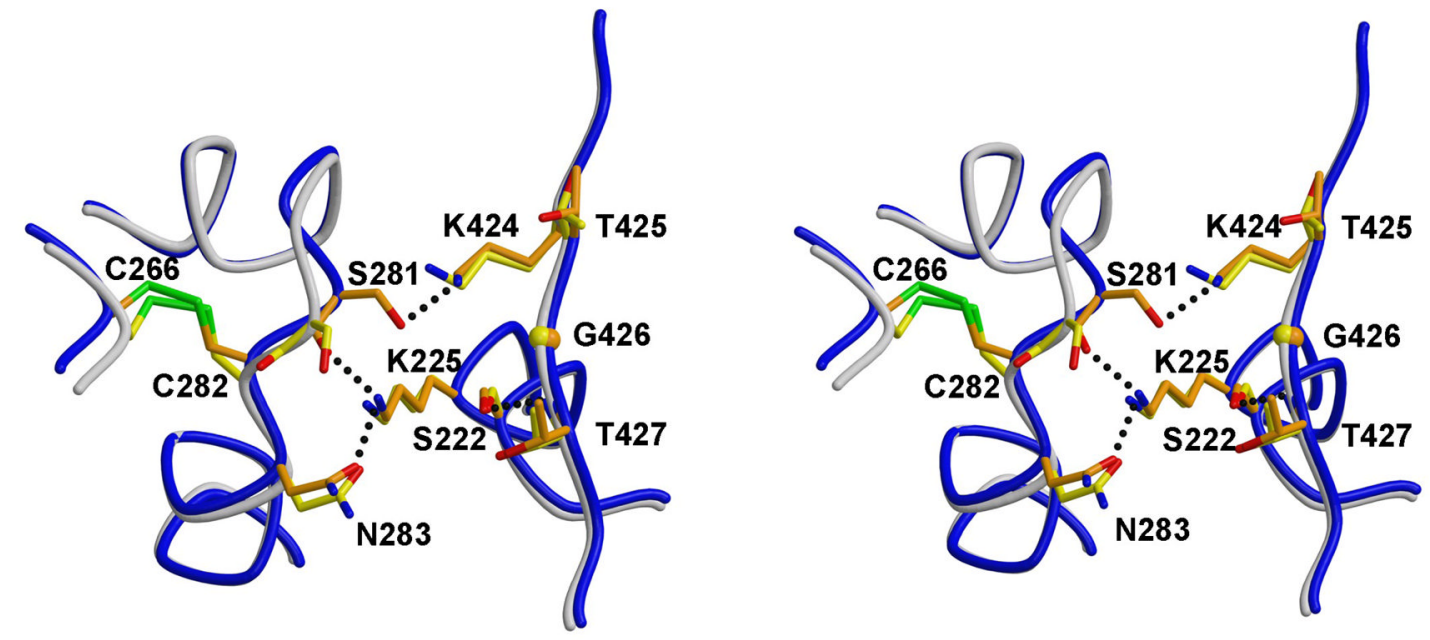

B
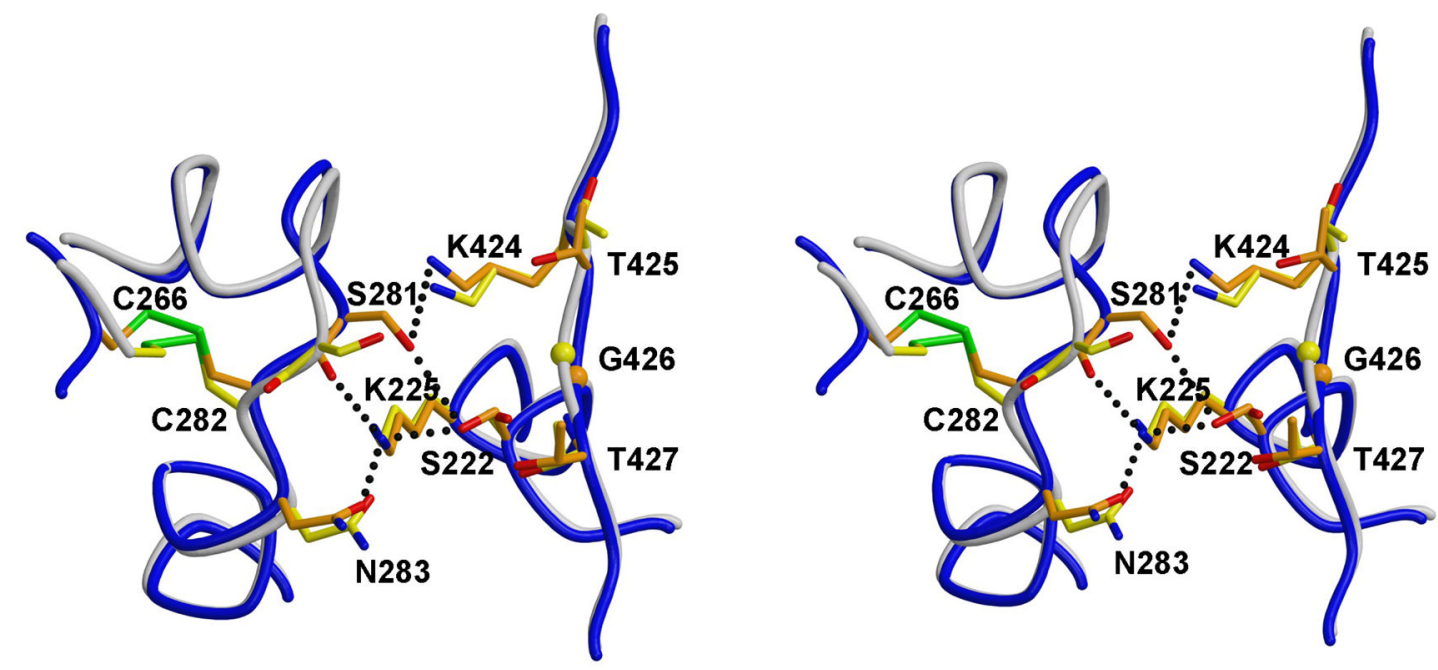

Fig. 2.

Superimposition of the two apo forms in the active site region showing the canonical position for the $\mathrm{SxN}$ motif in the new structure of PBPA. In this stereoview, the previous apo structure (His-tagged) is displayed as a grey backbone with yellow bonds, and the new apo structure is a blue backbone with orange bonds. In the latter, Ser281 of the SxN motif now lies in a canonical position and mediates hydrogen bonds with Lys424 (dashed lines). Only molecule A of the asymmetric unit is shown, but the shift is very similar in molecule B. 


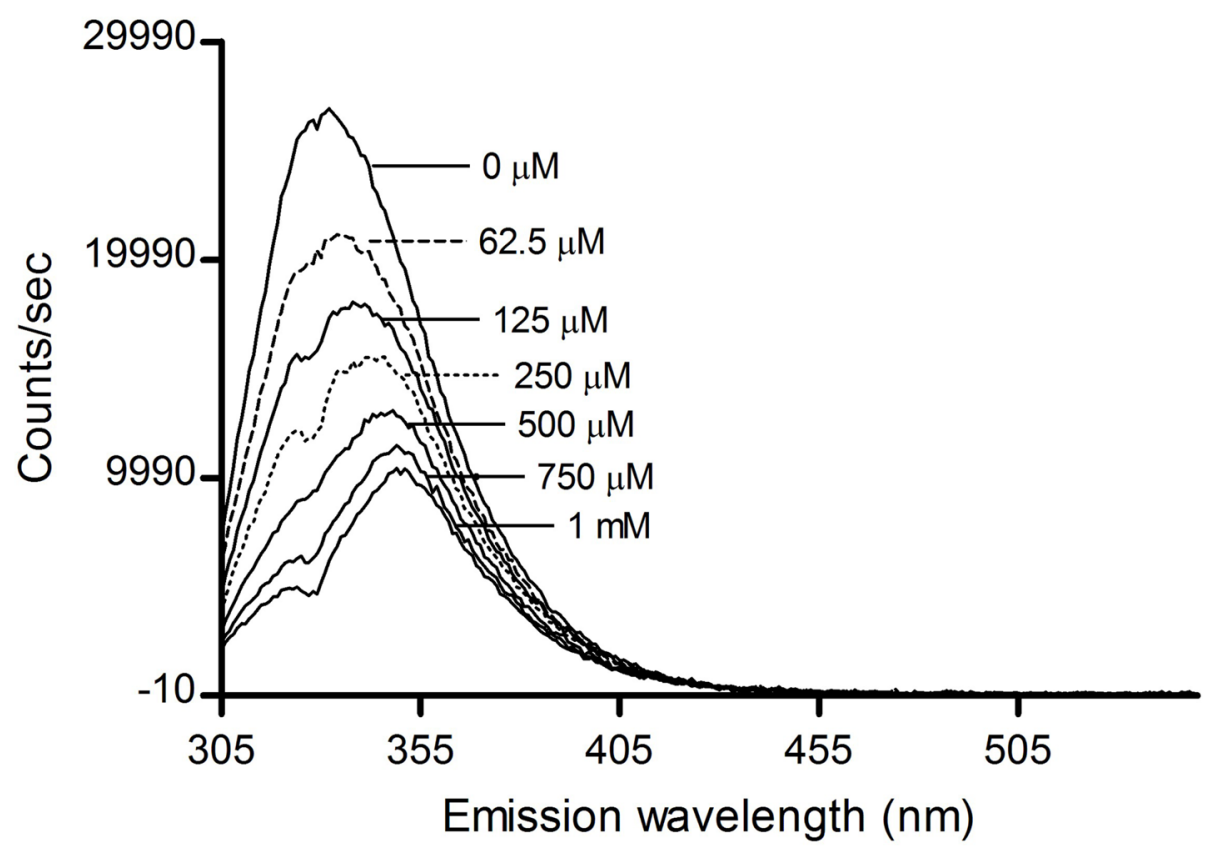

Fig. 3.

Intrinsic fluorescence emission scans of PBPA $(0.5 \mu \mathrm{M})$ following excitation at $292 \pm 2 \mathrm{~nm}$ and titrated with increasing concentrations of penicillin $\mathrm{G}$ from 0 to $1 \mathrm{mM}$. Fluorescence emission shows increasing quenching and shift of the maximum as acylation of the PBP increases. 


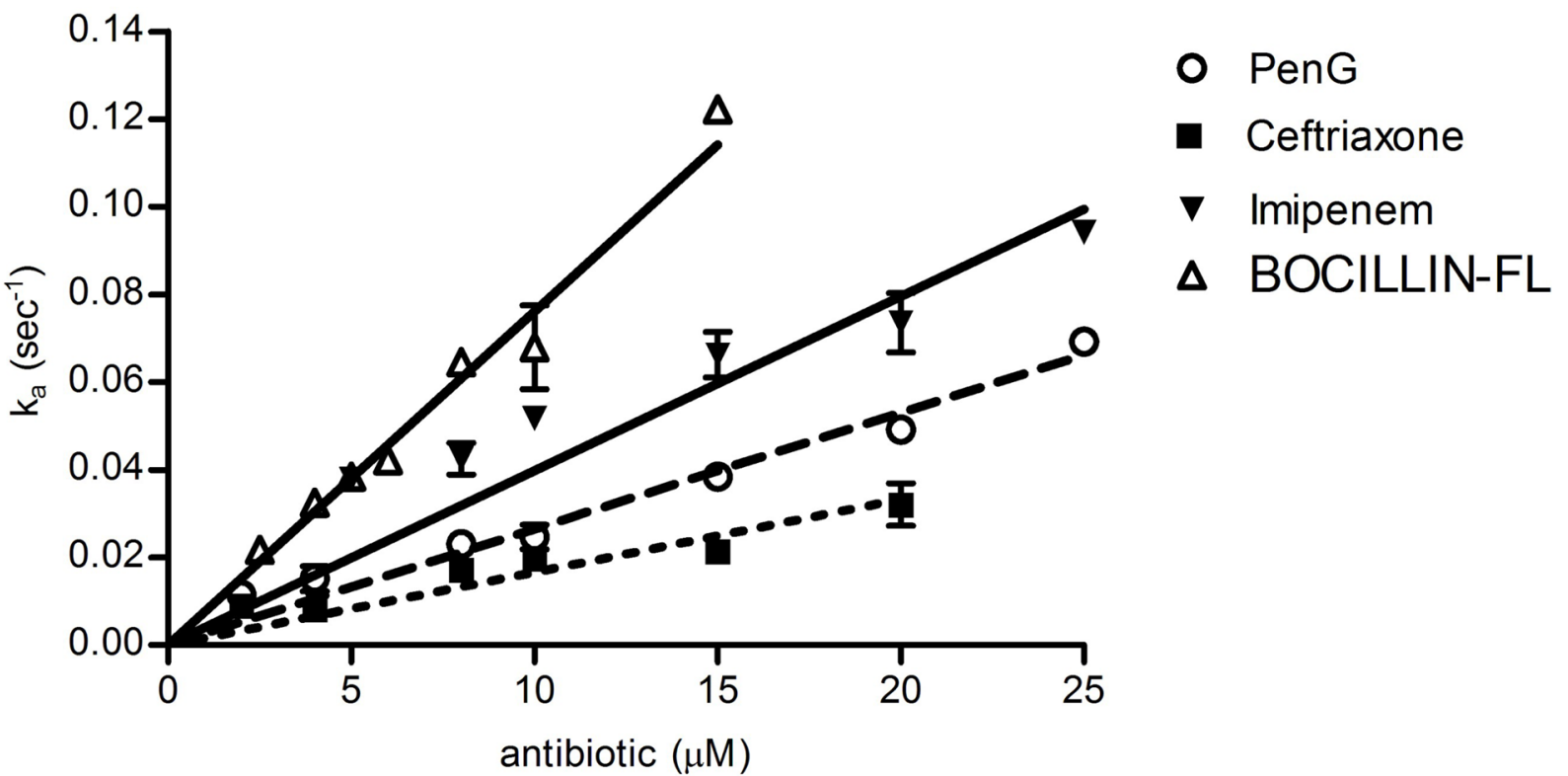

Fig. 4.

Determination of second-order acylation rate constants for the interaction between PBPA and $\beta$-lactam antibiotics using data measuring intrinsic fluorescence. Second order rate constants $\left(k_{2} / K_{S}\right)$ were derived from plot of $k a$ vs [S], where $k_{2} / K_{S}$ corresponds to the slope of the line. 
A

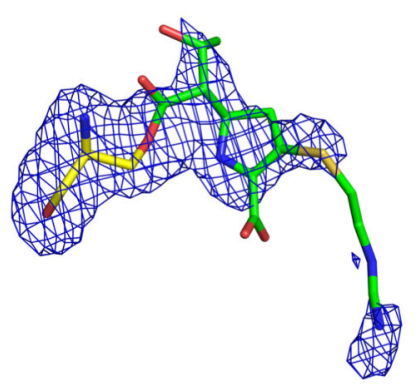

B

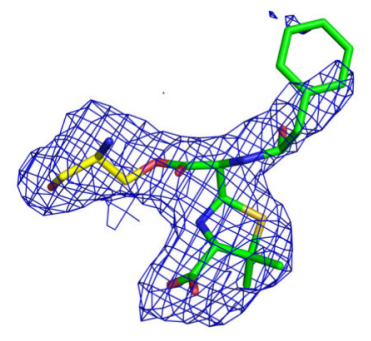

C

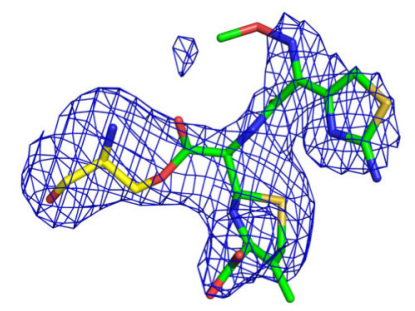

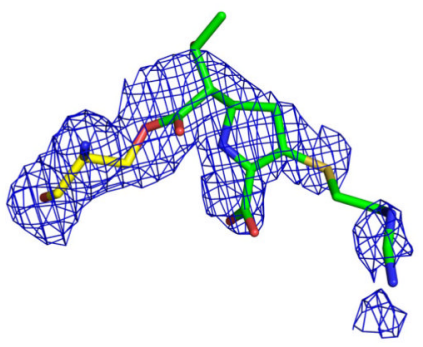
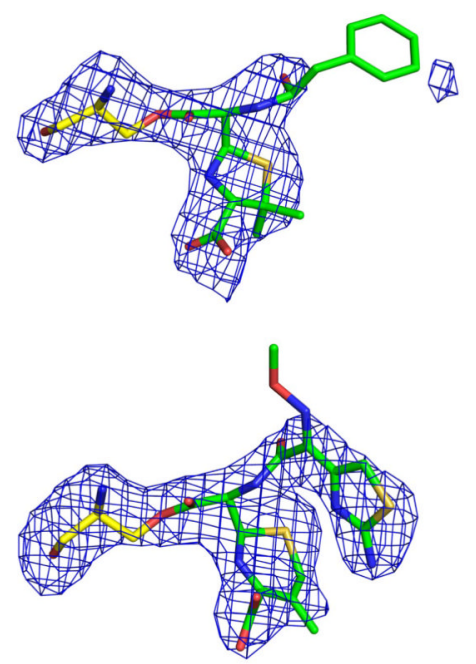

Fig. 5.

Electron density of antibiotics in the acylated structures of PBPA. In each case, $|F o|-|F c|$ difference electron density is shown, calculated after a refinement in which the occupancy of the atoms corresponding to each $\beta$-lactam and Ser222 were set to zero. A. imipenem contoured at $2.5 \sigma$. B. penicillin $\mathrm{G}$, contoured at $2.8 \sigma$. C. ceftriaxone, contoured at $2.5 \mathrm{~s}$. Density for both molecules of the asymmetric unit are shown, with molecule A on the left and molecule B on the right. In all cases, Ser222 is colored with yellow bonds and the antibiotic is colored with green bonds. 
A
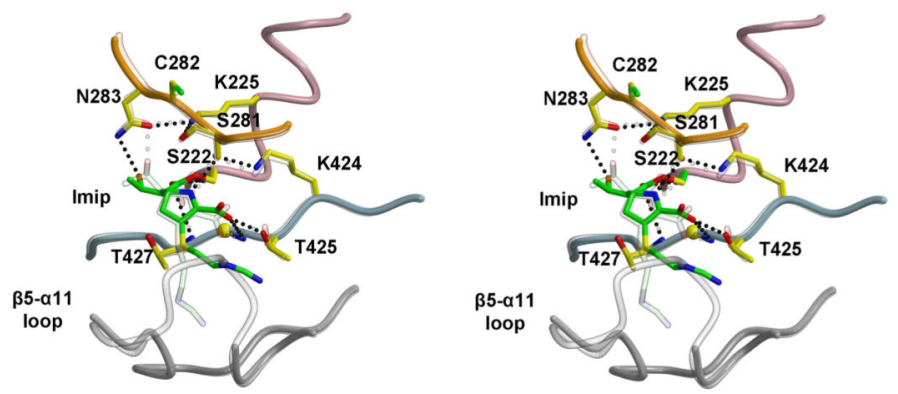

B
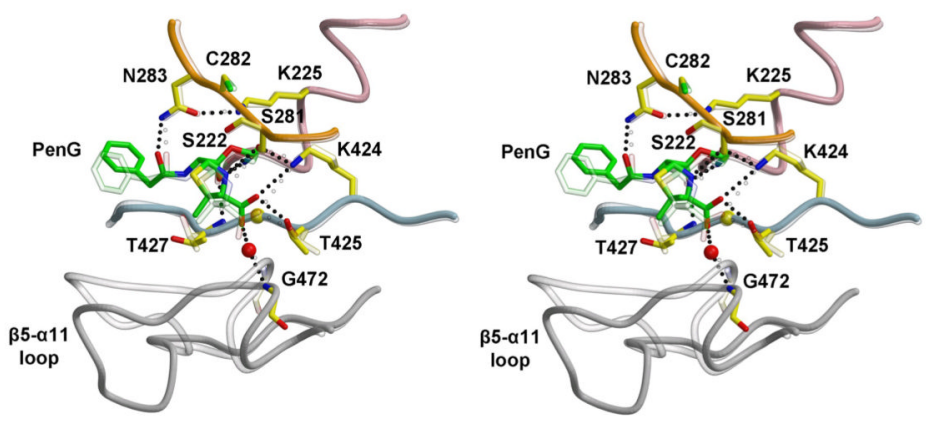

C
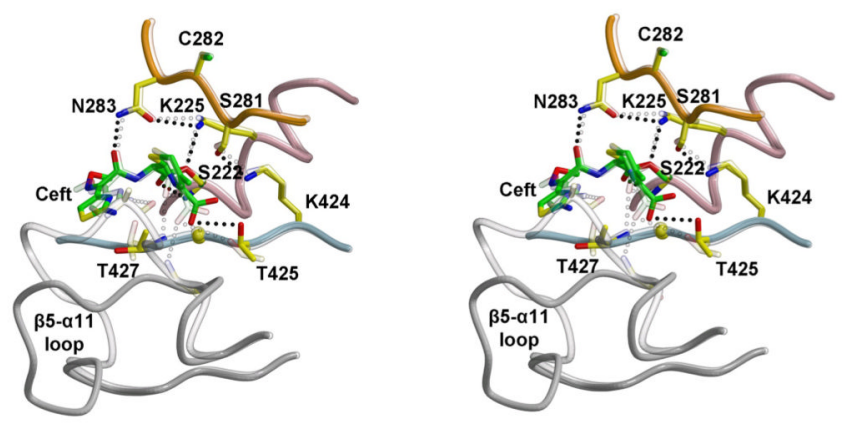

Fig. 6.

Contacts made by each antibiotic and position of the $\beta 5-\alpha 11$ loop in acylated structures of PBPA. Each panel shows a stereo view of the active site after superimposition of molecule A with molecule B of the asymmetric unit, where the latter is rendered with transparency. The $\mathrm{C} \alpha$ backbone is colored as follows: $\beta 3$ containing the KTG motif is blue, $\alpha 2$ containing the SxxK motif is pink, the $\alpha 4-\alpha 5$ loop containing the SxN motif is orange, and the $\beta 5-\alpha 11$ loop is grey. Selected residues are displayed with yellow bonds and each antibiotic is displayed with green bonds. Potential hydrogen bonds are shown as dashed lines. A, imipenem; $\mathbf{B}$, penicillin $\mathrm{G}$, and $\mathbf{C}$, ceftriaxone. 

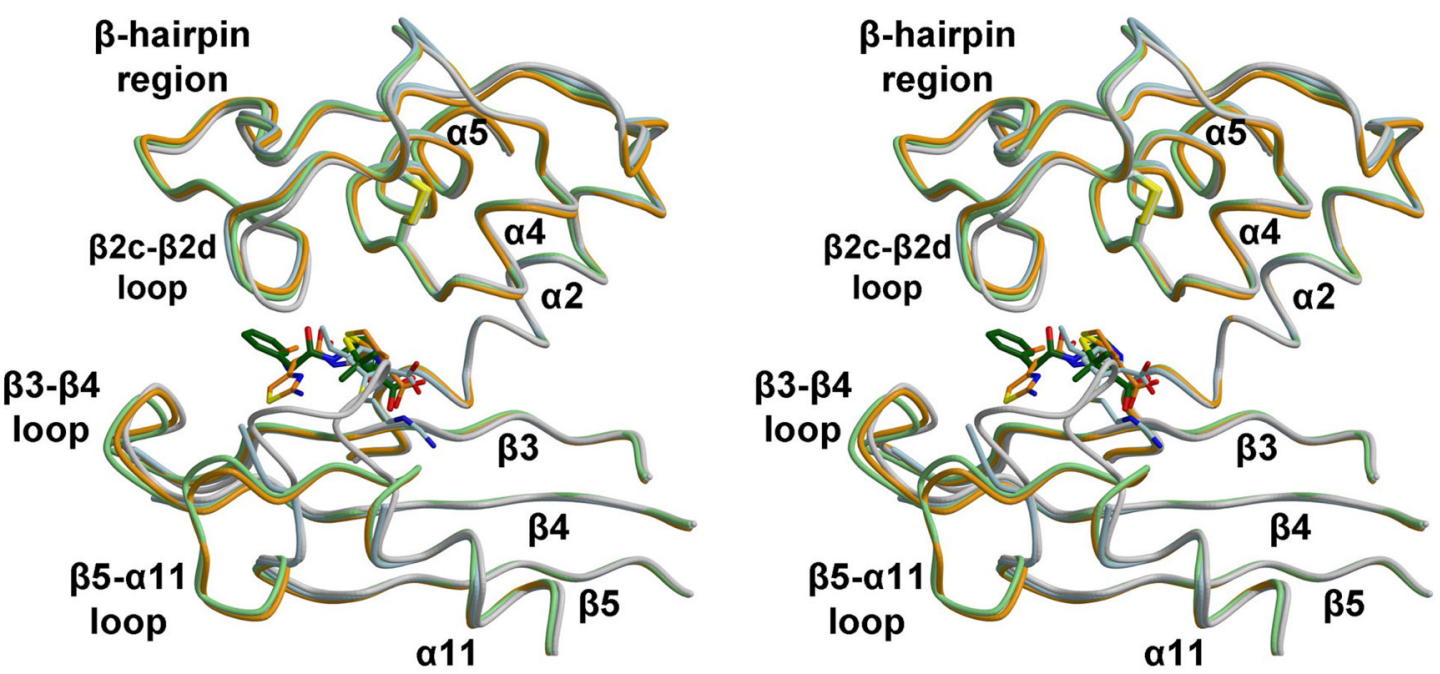

B
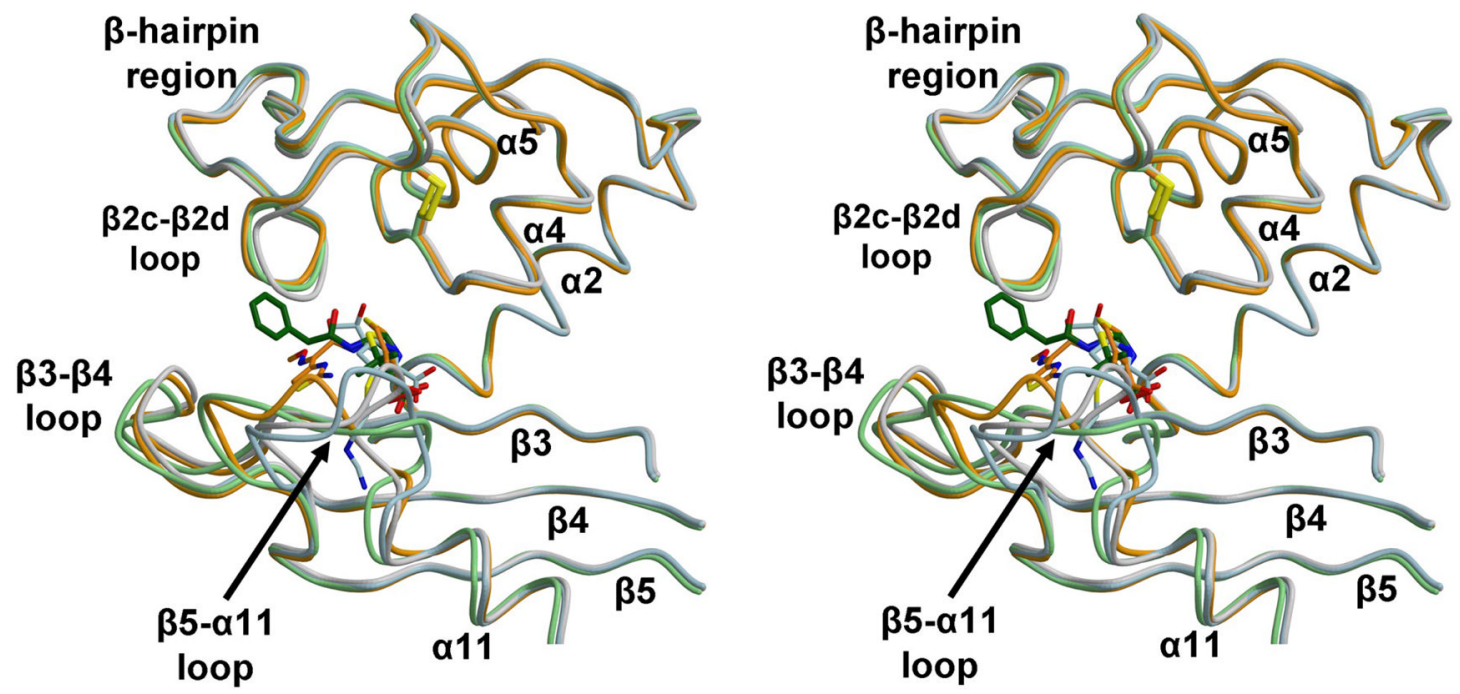

Fig. 7.

Superimposition of the active sites of PBPA and of the three acylated structures. Each structure is displayed as a $\mathrm{C} \alpha$ backbone where apo PBPA is grey, the complex with imipenem is blue; that for penicillin $\mathrm{G}$ is green and that for ceftriaxone is orange. Imipenem, penicillin $\mathrm{G}$ and ceftriaxone are also shown in bonds form with corresponding colors.

Elements of secondary structure are labeled according to ${ }^{10}$ and the disulfide bridge between the $\beta$-hairpin region and the $\mathrm{SxN}$ motif is also shown. Superimpositions of both molecules of the asymmetric unit are shown: A, molecule A and $\mathbf{B}$, molecule B. 


\section{Table I}

Data collection and refinement statistics

\begin{tabular}{|c|c|c|c|c|}
\hline Antibiotic & Apo & Imipenem & Penicillin G & Ceftriaxone \\
\hline Molarities of soak (mM) & - & 16 & 100 & 100 \\
\hline Time of soak (hrs) & - & 5 & 3 & 2 \\
\hline Wavelength $(\AA)$ & 1.00 & 1.0 & 1.0 & 1.0 \\
\hline Resolution range $(\AA)$ & $\begin{array}{l}46.1-2.0(2.03- \\
2.00)\end{array}$ & $\begin{array}{l}39.0-2.2(2.28- \\
2.20)\end{array}$ & $\begin{array}{c}44.3-2.3(2.38- \\
2.30)\end{array}$ & $\begin{array}{c}45.7-2.4(2.49- \\
2.40)\end{array}$ \\
\hline $\operatorname{Rmerge}^{l}(\%)$ & $11.8(70.6)$ & $8.4(55.2)$ & $8.0(49.7)$ & $8.0(62.3)$ \\
\hline Redundancy & $12.4(6.2)$ & $14.9(8.8)$ & $10.9(8.6)$ & $10.2(7.3)$ \\
\hline Completeness $(\%)$ & $99.9(99.3)$ & $99.3(96.1)$ & $98.1(91.1)$ & $98.7(89.6)$ \\
\hline$\langle\mathrm{I}\rangle \mid\langle\sigma \mathrm{I}\rangle$ & $35.4(2.1)$ & $40.3(2.5)$ & $34.0(2.7)$ & $34.5(2.2)$ \\
\hline Number of water molecules & 193 & 58 & 48 & 21 \\
\hline Crystallographic R factor (\%) & 20.5 & 21.6 & 19.7 & 20.3 \\
\hline $\mathrm{R}$ work (\%) & 20.4 & 21.4 & 19.5 & 20.1 \\
\hline $\mathrm{R}$ free $(\%)$ & 23.5 & 25.7 & 24.3 & 24.7 \\
\hline \multicolumn{5}{|l|}{ RMS deviations from ideal stereochemistry:- } \\
\hline bond lengths $(\AA)$ & 0.010 & 0.011 & 0.011 & 0.010 \\
\hline bond angles $\left(^{\circ}\right)$ & 1.30 & 1.33 & 1.35 & 1.36 \\
\hline \multicolumn{5}{|l|}{ Mean B factors $\left(\AA^{2}\right)$ : } \\
\hline all atoms & 37.5 & 44.6 & 46.6 & 53.0 \\
\hline main chain & 36.5 & 43.8 & 45.8 & 52.3 \\
\hline side chain \& waters & 38.6 & 45.5 & 47.6 & 53.8 \\
\hline antibiotic & - & 66.9 & 53.5 & 64.3 \\
\hline \multicolumn{5}{|l|}{ Ramachandran plot: } \\
\hline$\%$ residues in most favored region & 91.6 & 92.0 & 90.1 & 90.3 \\
\hline$\%$ residues in additionally allowed regions & 7.5 & 7.0 & 9.1 & 8.8 \\
\hline$\%$ residues in generously allowed regions & 0.6 & 0.7 & 0.4 & 0.7 \\
\hline$\%$ residues in disallowed regions & 0.3 & 0.3 & 0.4 & 0.1 \\
\hline $\begin{array}{l}\mathrm{RMSD}^{l} \text { of } \mathrm{C} \alpha \text { atom with native structure }(\AA) \\
(\text { molecule } \mathrm{A} / \text { molecule } \mathrm{B})\end{array}$ & - & $0.42 / 0.62$ & $1.18 / 0.76$ & $1.25 / 0.79$ \\
\hline PDB code & $3 \mathrm{UN} 7$ & 3UPN & $3 \mathrm{UPO}$ & 3UPP \\
\hline
\end{tabular}

${ }^{1}$ Superimposition performed using all common main chain atoms in the residue range 50-488. All atoms were refined with an occupancy of 1. 
Table II

Kinetic parameters for the interaction between PBPA and $\beta$-lactam antibiotics

\begin{tabular}{|c|c|c|}
\hline \multirow{2}{*}{$\boldsymbol{\beta}$-lactam } & \multicolumn{2}{|c|}{ Second-order rate constants of acylation, $\boldsymbol{k}_{\mathbf{2}} / \boldsymbol{K}_{\boldsymbol{S}}\left(\mathbf{M}^{\mathbf{- 1}} \mathbf{s e c}^{\mathbf{- 1}}\right)$} \\
\cline { 2 - 3 } & \multicolumn{2}{|c|}{ Method } \\
\hline Bocillin-FL & $7,610 \pm 250$ & SDS-PAGE \\
\hline Penicillin G & $2,650 \pm 100$ & $7,100 \pm 430$ \\
\hline Ceftriaxone & $1,660 \pm 100$ & $2,860 \pm 130$ \\
\hline Imipenem & $3,980 \pm 180$ & $1,680 \pm 100$ \\
\hline
\end{tabular}

Data values represent mean \pm SEM (standard error of the mean) 